Langages, septembre $1980, n^{\circ} 59$, Conduites langagières et sociolinguistique scolaire, sous la direction de F.François.

79

Rhian JONES, Marie-Christine POUDER

Université de Paris-V

\title{
LES ÉCHANGES ADULTE-ENFANT EN SITUATION SCOLAIRE
}

On voudrait ici apporter quelques résultats tirés d'une enquête que nous avons menée (Université René Descartes et DGRST) dans sept classes de la région parisienne. Dans chaque enquête, on peut toujours poser la question de la représentation du corpus. On ne peut jamais la résoudre directement. Disons seulement que la relative constance des résultats que nous avons obtenus dans ces sept classes tend à indiquer qu'il ne s'agit pas d'un phénomène aléatoire ...

En fait, on ne présente ici que les résultats obtenus dans cinq classes. On verra en effet que nous voulions à la fois contraster les types de pédagogie et les types de contenu ; mais il n'était pas question d'obliger les enseignants à présenter tel ou tel contenu. C'est seulement dans cinq classes que nous avons pu opposer enseignement de la grammaire et du vocabulaire.

Bon nombre d'analyses du discours enfantin, voire même adulte, ont permis de conclure que toute production linguistique dépend étroitement de la situation de communication dans laquelle l'émetteur se trouve. Deux variables se révèlent particulièrement déterminantes pour le type de langage produit en situation de dialogue : l'interlocuteur et le contenu véhiculé. Les études en question permettent de constater que selon certains critères (types de structures syntaxiques utilisées ou fréquence relative d'emploi de ces structures par exemple), les ressemblances entre les performances d'enfants sont aussi importantes que les divergences, et cela nonobstant l'appartenance sociale des parents de l'enfant. Autrement dit, placer des enfants dans une situation de communication identique quant au contenu et à l'interlocuteur les amène à produire un langage dont la forme frappe bien davantage par son homogénéité que par sa diversité, et cela en dépit des différences de l'appartenance sociale des parents. En outre, les types de productions enfantines émises dans telles ou telles circonstances se rapprochent à un point tel qu'elles se démarquent de manière considérable de productions faites par les mêmes enfants dans d'autres situations (on ne répond pas au médecin comme on dialogue avec le voisin). Ce qui amène certains linguistes à parler de « compétences linguistiques » (aptitude à parler dans telle ou telle situation) plutôt que d'une compétence linguistique globale. Toute analyse du langage enfantin donc (à ne s'en tenir qu'au discours des enfants) qui pousserait à des comparaisons 
globales (décompte de structures dites complexes par exemple) et qui ferait abstraction de la variable «situation de communication » dans laquelle le langage est produit, ferait preuve de graves défaillances théoriques.

\section{Déroulement de l'enquête}

L'hypothèse qui sous-tend le travail ci-dessous présenté est que le dialogue oral scolaire adulte-enfant en salle de classe fonctionne, lui aussi, avec des lois et des codes spécifiques, le but de cette étude étant de cerner ce fonctionnement dans un

\section{0}

maximum de dimensions : participation verbale de l'élève en cours, structures syntaxiques utilisées, contenu du cours, type de questions posées par le maître par exemple. L'analyse porte sur des productions orales de 111 enfants enregistrés pendant deux cours de français dans cinq écoles primaires publiques (niveau CMI) de la région parisienne. Cinq instituteurs, dont trois affirmaient pratiquer une pédagogie «libre », dispensaient auprès de leur classe habituelle un cours de vocabulaire et un cours de grammaire. Dans tous les cas il s'agit d'un type d'enseignement où le maître s'adresse à l'ensemble des élèves qui lui accordent apparemment leur attention, non pas d'un enseignement où les élèves sont réunis en sous-groupes pour travailler. Il s'agit également de maîtres de l'enseignement public très expérimentés ayant un minimum de sept ans de pratique (et observés seulement après « sélection » de la part de l'IDEN).

Les élèves, quant à eux, se trouvent dans des classes à composition mixte du point de vue de la catégorie socioprofessionnelle de leurs parents. Ils sont tous individualisés au cours de l'étude : on étudie aussi bien les prises de parole de chaque participant que les phénomènes globaux caractéristiques de la participation de l'ensemble du groupe «élèves ». A noter particulièrement que par opposition à certaines études, les productions linguistiques des enfants ne sont pas relevées en situation de test ou en entretien individuel, mais dans une situation de groupe où ils sont confrontés à un interlocuteur privilégié, leur maître, et aussi à des enfants d'un milieu culturel d'origine différent du leur.

La présentation qui suit met en évidence les aspects quantitatifs (degré de la participation des élèves au cours) et syntaxiques (type de structures prédominantes et fréquence d'emploi) en donnant une attention particulière dans les deux cas à la variable « appartenance sociale de l'enfant », mesurée par la profession des parents.

\section{Quelques résultats}

Si l'analyse menée jusqu'ici permet de cerner certains traits caractéristiques du type d'échange produit en milieu scolaire en général (par exemple dans toutes les leçons observées, le nombre total d'interventions attribuables à chaque enfant a 
varié de façon considérable), force est de constater néanmoins que les caractéristiques des cours de vocabulaire et celles des séances de grammaire sont telles qu'il convient de distinguer pour cette étude deux situations linguistiques différenciées (I'ensemble des cours de vocabulaire vs l'ensemble des cours de grammaire). Par exemple, la distribution des prises de parole parmi les effectifs présents est pratiquement constante pour chaque situation. Si I'on regroupe par trois tranches les effectifs d'élèves présents à chaque leçon, le pourcentage d'interventions attribué au tiers d'élèves qui parle le moins dans les cours est de $9 \%$ en moyenne en grammaire, et de $3 \%$ en vocabulaire ; chez le tiers d'élèves qui parle le plus, ces pourcentages sont respectivement de $61 \%$ et de $80 \%$. Autrement dit, le vocabulaire rend plus aiguë encore la tendance constatée en grammaire quant à la disproportion entre les distributions des prises de parole des enfants. Qui plus est, les écarts autour des moyennes citées sont faibles, ce qui permet de conclure à une remarquable uniformité pour ce critère sur cinq instituteurs différents et cinq établissements différents. Etant donnée la régularité avec laquelle ces résultats se confirment pour chaque séance observée (en dépit de l'utilisation de techniques ou de manuels différents), il conviendrait même de parler d'un certain déterminisme quant aux possibilités pour un élève d'accéder à la parole dans un cours de vocabulaire ou de grammaire.

\section{1}

Devant ce déterminisme au niveau de l'ensemble des groupes d'élèves, nous avons essayé de voir si, au niveau des individus, le changement de situations a permis à d'aucuns d'augmenter ou de diminuer de façon significative le pourcentage de leurs contributions verbales par rapport aux autres membres du groupe. Or $9 \%$ des élèves seulement modifient de manière considérable leur taux de participation relatif en passant du premier au troisième tiers des élèves ou inversement. $49 \%$ des élèves en moyenne modifient à peine leur statut : $20 \%$ des élèves dans le premier tiers en grammaire y restent en vocabulaire ; $16 \%$ se situant dans le troisième tiers en grammaire $s^{\prime} y$ retrouvent en vocabulaire. Pour ce dernier critère, l'étude de l'origine socioculturelle est significative. $29 \%$ des enfants issus du milieu aisé se trouvent dans le tiers qui parle le plus dans les deux situations (contre $12 \%$ pour le milieu non favorisé), alors que l'on constate la tendance inverse pour ceux. qui parlent le moins : $8 \%$ des «bourgeois » présents se trouvent dans le $3^{\text {ème }}$ tiers dans les deux situations, contre $21 \%$ en ce qui concerne les élèves de milieu non favorisé.

Au niveau de l'ensemble du groupe d'élèves, l'identification des intervenants selon leur milieu socioculturel d'origine permet de constater que, dans les classes où les élèves des deux milieux identifiés pour cette étude sont numériquement égaux, à peu de choses près ce sont les enfants de milieu favorisé qui fournissent la majorité des prises de parole, majorité qui devient nettement plus élevée dans le cours de vocabulaire (sauf dans le cas d'une classe où le phénomène inverse est observé). Chez le $5^{\text {ème }}$ instituteur, où les enfants favorisés sont très nettement minoritaires (4 sur 23 élèves présents) ils ne fournissent pas moins de $43 \%$ de l'ensemble des interventions « élèves ». Dans chaque cas, quels que soient les effectifs appartenant à un groupe social donné dans une classe, nous constatons la même 
tendance : la grande majorité des prises de parole attribuées à chaque groupe, dans chaque situation est le fait de $50 \%$ seulement des enfants présents appartenant à ce milieu. Cette importante variation intragroupe social va dans le même sens pour les deux groupes sociaux distingués et pour les deux situations. Pour ce critère, et même pour les autres phénomènes observés au niveau des prises de parole en situation de groupe, on peut se demander s'il s'agit ici de phénomènes généraux caractéristiques du discours en milieu scolaire, ou bien s'il s'agit de traits caractéristiques de n'importe quelle situation de conversation en groupes à plusieurs participants.

La régularité des résultats obtenus au niveau des prises de parole pour chaque situation se constate également au niveau des phénomènes syntaxiques. Une étude globale du type de structures utilisées et de leur fréquence relative d'emploi permet de conclure à la prédominance massive de réponses par syntagmes (nominaux pour la plupart) dans les classes de grammaire, consacrées pour l'essentiel à une activité d'analyse grammaticale. Par contre, les cours de vocabulaire donnent lieu à des productions linguistiques plus longues et plus variées au niveau du discours de l'enfant (exception faite d'une classe). Sont utilisés surtout des énoncés verbaux ou énoncés avec actualisateur.

La comparaison entre groupes sociaux pour chaque situation différencie les deux situations de la manière suivante. Alors qu'en grammaire les variations syntaxiques entre enfants de milieux différenciés sont minimes (les enfants issus d'un milieu défavorisé s'expriment systématiquement de la même façon que les enfants aisés) il en va tout autrement pour le vocabulaire où l'on constate que les énoncés verbaux seraient bien davantage le fait de la «bourgeoisie », alors que les interventions par syntagmes sont fournies par le milieu non favorisé (et ceci à partir des questions précises des maîtres).

L'étude des prises de parole a déjà mis en évidence la prédominance des enfants de milieu favorisé en vocabulaire. Sur ce point, on trouve corrélativement chez eux, dans la même situation, une forte prépondérance de l'utilisation d'énoncés verbaux et

82

non de réponses par syntagmes. On constate ici une situation où sont induites des divergences syntaxiques parmi les groupes sociaux. On a en effet :

Moyenne des maîtres

Moyenne des I (favorisés)

Moyenne des II (non favorisés)

Moyenne des élèves
1

2

49,44

47,40

35,44

41,42
15,38

14,14

13,33

13,73
3

31,04

35,41

48,57

41,99
4 5

$\begin{array}{ll}4,12 & 23,03 \\ 3,15 & 21,30 \\ 2,87 & 17,27 \\ 3,01 & 19,28\end{array}$


(1: verbes conjugués ; 2 : actualisateurs ; 3 : non verbaux ; 4 : oui/non; 5 : prédicatoïdes.)

En fait, il y a beaucoup plus de différence, du moins dans cette situation particulière, entre les enfants issus de milieux différents qu'entre le maître et les enfants du milieu I, ces derniers dialoguant finalement de manière privilégiée en utilisant un langage aux structures très proches ; faudrait-il ici interroger plus précisément les origines sociales du maître (cf. enquête d'Ida BERGER) ?

En revanche, en grammaire, les différences entre le comportement verbal des deux groupes sociaux se situent, non pas au niveau de l'utilisation de structures syntaxiques, mais au niveau des stratégies d'intervention employées par les enfants.

Par rapport aux enfants de milieu défavorisé, les enfants du milieu aisé ont tendance à consacrer une plus grande partie de leur discours à des initiatives (exception faite d'une classe), à intervenir sans attendre que leur maître les nomme, et à parler immédiatement après un autre élève, sans que soit interposée une intervention du maître.

Ainsi, sous une forme quantitative, on obtient les totaux suivants en grammaire et en vocabulaire ainsi que les comparaisons entre les maîtres 1 et 3 d'une part, 4 et 5 de l'autre, de notre échantillon, regroupés pour manifester leur contraste :

Grammaire

\begin{tabular}{|l|l|l|l|l|}
\hline GRAMMAIRE & $\begin{array}{l}\text { Interventions } \\
\text { des élèves } \\
\text { après discours } \\
\text { du maître }\end{array}$ & $\begin{array}{l}\text { Interventions } \\
\text { des élèves } \\
\text { après un élève }\end{array}$ & $\begin{array}{l}\text { Appel } \\
\text { nominal }\end{array}$ & $\begin{array}{l}\text { Nombre } \\
\text { d'initiatives }\end{array}$ \\
\hline TOTAL & 1021 & 102 & 690 & 148 \\
\hline Maîtres 1 et 3 & $90,92 \%$ & $9,08 \%$ & $61,64 \%$ & $13,18 \%$ \\
\hline Maîtres 1 et 3 & $95,45 \%$ & 32 & 493 & 72 \\
& 350 & $4,55 \%$ & $70,13 \%$ & $10,24 \%$ \\
\hline & $83,33 \%$ & 70 & 197 & 76 \\
\hline
\end{tabular}

Vocabulaire

\begin{tabular}{|l|l|l|l|l|}
\hline TOTAL & 1010 & 298 & 360 & 392 \\
& $77,22 \%$ & $22,78 \%$ & $27,52 \%$ & $29,97 \%$ \\
\hline Maîtres 4 et 5 & 645 & 106 & 282 & 189 \\
& $85,88 \%$ & $14,11 \%$ & $37,55 \%$ & $25,70 \%$ \\
\hline Maîtres 4 et 5 & 365 & 192 & 78 & 203 \\
& $65 ? 53 \%$ & $34,47 \%$ & $14 \%$ & $36,44 \%$ \\
\hline
\end{tabular}


Dans cette situation où joue à la fois la matière enseignée et les caractéristiques propres à chaque enseignant, on constate les variations suivantes entre enfants en fonction de leur milieu d'origine chez les deux maîtres les plus différents, 1 et 4 :

Grammaire

\begin{tabular}{|l|l|l|l|l|}
\hline & $\begin{array}{l}\text { Interventions } \\
\text { des èlèves } \\
\text { après discours } \\
\text { du maître }\end{array}$ & $\begin{array}{l}\text { Interventions } \\
\text { des élèves } \\
\text { après un élève }\end{array}$ & Appel nominal & $\begin{array}{l}\text { Nombre } \\
\text { d'initiatives }\end{array}$ \\
\hline $\begin{array}{l}\text { Maître 1 } \\
\text { Groupe 1 }\end{array}$ & $96,11 \%$ & $3,88 \%$ & $65,55 \%$ & $26,11 \%$ \\
Groupe 2 & $95,45 \%$ & $4,54 \%$ & $75,75 \%$ & $3,78 \%$ \\
\hline Maître 2 & & & & \\
Groupe 1 & $78,61 \%$ & $21,38 \%$ & $35,84 \%$ & $23,27 \%$ \\
Groupe 2 & $86,04 \%$ & $13,75 \%$ & $58,91 \%$ & $14,72 \%$ \\
\hline
\end{tabular}

Vocabulaire

\begin{tabular}{|l|l|l|l|l|}
\hline Maître 1 & & & \\
Groupe 1 & $84,81 \%$ & $15,18 \%$ & $31,93 \%$ & $28,79 \%$ \\
Groupe 2 & $88,07 \%$ & $11,92 \%$ & $42,20 \%$ & $6,55 \%$ \\
\hline Maître 4 & & & & \\
Groupe 1 & $60,07 \%$ & $39,92 \%$ & $11,30 \%$ & $27,20 \%$ \\
Groupe 4 & $66,66 \%$ & $33,33 \%$ & $28,07 \%$ & $19,29 \%$ \\
\hline
\end{tabular}

On peut constater (sur ces exemples comme ailleurs) :

a) en grammaire, lorsqu'il y a des interventions suivant des interventions d'autres élèves (classe 4) elles sont surtout le fait d'élèves des groupes favorisés ;

b) dans toutes les classes ce sont les enfants du milieu 2 (non favorisé) qui sont le plus l'objet d'appels nominatifs : il n'y a donc aucune ségrégation manifeste à leur égard ;

c) la tendance est à avoir un plus grand nombre d'énoncés-initiatives de la part des enfants de milieu favorisé ;

d) en vocabulaire, la tendance à la domination des enfants de milieu favorisé dans les prises de parole après les autres élèves se manifeste le plus souvent (sauf dans la classe 3) ; il est vrai que le nombre total de ces interventions étant faible I'interprétation en est difficile ; 
e) les interventions nominatives des maîtres sont en relation avec les énoncés dirigés, les non-nominatives avec les initiatives; les deux se recoupent avec les classes sociales ;

f) cette tendance générale se combine avec des variations importantes selon les maîtres : tout se passe comme si la prégnance de la situation et du contenu l'emportait en grammaire et qu'en revanche une part plus grande d'initiative des élèves et corrélativement d'énoncés des élèves suivant les énoncés des élèves et non ceux du maître étaient possibles, mais non nécessaires dans le cas du vocabulaire.

Ces variations se combinent régulièrement. Ainsi les maîtres 1 et 2 induisent la quasi totalité des énoncés en grammaire, induction qui reste dominante quoique moins forte chez les maîtres 4 et 5 . Cette induction, en particulier sous forme d'appels nominatifs, diminue beaucoup chez ces derniers maîtres, en vocabulaire,

\section{4}

puisque les appels non nominaux y constituent la grande majorité alors qu'ils étaient très faiblement majoritaires en grammaire (cette analyse devrait évidemment être complétée par une analyse de la communication non linguistique, l'appel personnel pouvant se faire par le regard ou le geste).

A partir de ces constatations, il n'y aurait qu'un pas à raire pour suggérer que l'exercice d'une pédagogie dite libre serait nettement favorable (dans le court terme tout au moins) aux enfants de milieu favorisé. On pourrait néanmoins avancer l'hypothèse inverse, à savoir que dans une classe, plus les élèves de milieu aisé ont la possibilité de prendre la parole de leur propre chef, plus les autres enfants le feront également.

De toute façon, les pourcentages de telles initiatives sont ici extrêmement réduits. Les cours de grammaire ne laissent qu'à peine place aux initiatives ( $5 \%$ en moyenne sur l'ensemble des cours observés) : les données à disposition sont donc minimes.

Devant un phénomène aussi complexe que l'échange adulte-enfant en milieu scolaire, il serait prématuré de conclure quant aux explications possibles des variations entre les situations. La répartition plus équilibrée des prises de parole parmi l'ensemble des élèves et plus particulièrement la participation accrue des enfants provenant du milieu non favorisé, en grammaire par rapport au vocabulaire, serait peut-être due au fait qu'un cours de grammaire amène les élèves à revoir très souvent un certain contenu et que, par conséquent, la possibilité de fournir une réponse « acceptable » est ainsi offerte à un plus grand nombre d'élèves. Nous avançons également I'hypothèse d'un éventuel lien entre participation et contenu du cours dans la mesure où, à notre sens, l'analyse grammaticale est souvent un artefact scolaire, propre à cette institution, où le vécu de l'enfant a moins de poids, alors qu'en vocabulaire le poids de l'apport culturel et du vécu extrascolaire est autrement plus important, ce qui pourrait entraîner la 
conséquence constatée : les élèves favorisés participent plus activement au cours. Il se peut aussi que l'agencement des réponses par étapes successives, avec des questions exigeant une réponse précise, type de pédagogie appliquée en grammaire, soit favorable aux enfants du milieu socio-culturel défavorisé . Nous rappelons ici néanmoins les grandes variations intra-groupe social (participation très réduite de $50 \%$ des enfants de chaque groupe), pour invoquer une variable interactionnelle (souvent négligée dans certaines études sociologiques) qui dépasse toute explication uniquement en termes de classes sociales.

On retrouve des résultats similaires dans les types de questions utilisées par les maîtres. Nous avons pu les classer en quatre rubriques : questions lexicales (Comment ça s'appelle ?) ; ordres (Donnez la règle.) ; questions exprimées par un terme grammatical (Lequel ?) ; questions sous forme de « trous » (C'est un... ?).

Sur ce plan, les formulations préférentielles semblent varier avec les maîtres. On a globalement (en vocabulaire) 513 questions lexicales, 148 ordres, 115 questions portées par un terme grammatical et 103 énoncés à « trous ». Si les questions lexicales constituent un ensemble important (elles sont majoritaires chez quatre enseignants), elles ne constituent que $26,9 \%$ des interventions chez I'un d'entre eux. Les ordres sont peu fréquents chez trois maîtres, quasi absents chez le maître 5 , dominants chez le maître 4 ; les questions grammaticales sont fréquentes seulement chez les maîtres 1 et 5 . Enfin les «trou s» sont importants seulement chez le maître 4. C'est-à-dire que la forme précise de l'incitation n'est pas corrélée directement avec l'aspect plus ou moins directif du maître, même si globalement les questions lexicales et les ordres entraînent plus de réponses « ouvertes », les questions fermées et les «trous » plus de réponses strictement contraintes.

Si I'on se demande enfin (comme on s'est demandé qui ouvrait le discours) qui le ferme, on trouve que, comme la fonction d'ouverture, la fonction de clôture est jouée majoritairement par les maîtres. En moyenne, dans $80,66 \%$ des cas. Mais avec

d'importantes disparités : 89,35\% pour les maîtres 1, 2, 3 et seulement $68,94 \%$ pour les deux derniers. Majoritairement, il s'agit de reprises lexicales $(53,68 \%)$ des cas) : le maître clôt l'échange en reprenant le mot qui vient d'être dit. D'autre part, dans $76,87 \%$ des cas la reprise est favorable (approbation). Elle porte enfin sur le contenu beaucoup plus que sur la correction du discours. Comme précédemment, nous avons ici des traits qu'on peut considérer comme caractéristiques de la situation. En revanche, le plus souvent les reprises lexicales ou par une glose s'adressent à un enfant de milieu défavorisé alors que la réponse par anaphorique ou elliptique s'adresse plus à un enfant de milieu favorisé. Corrélativement, chez trois maîtres, les « refu s» sont plus nombreux à l'égard des « défavorisés ». On ne peut donc pas ranger l'ensemble des conduites verbales des maîtres sur un seul axe directif-non directif, non plus que globalement favorable ou défavorable aux enfants de milieu non favorisé. 
Evaluation des contributions des élèves par le maître. (Base : nombre d'interventions attribuées aux élèves suivies et reprises par le maître.)

\begin{tabular}{|c|c|c|c|c|c|}
\hline \multirow[t]{2}{*}{ Maîtres } & Groupes & \multicolumn{2}{|c|}{$\begin{array}{l}\text { Le maître est } \\
\text { favorable }\end{array}$} & \multicolumn{2}{|c|}{$\begin{array}{l}\text { Le maître modifie, } \\
\text { n'accepte pas }\end{array}$} \\
\hline & $\mathrm{N}$ & $\mathrm{N}$ & $\%$ & $\mathrm{~N}$ & $\%$ \\
\hline 1 & $\begin{array}{lr}\text { Total } & 220 \\
\text { G1 } & 133 \\
\text { G2 } & 87\end{array}$ & $\begin{array}{r}166 \\
101 \\
65\end{array}$ & $\begin{array}{l}75,45 \\
75,94 \\
74,71\end{array}$ & $\begin{array}{l}54 \\
32 \\
22\end{array}$ & $\begin{array}{l}24,54 \\
24,06 \\
25,29\end{array}$ \\
\hline 2 & $\begin{array}{lr}\text { Total } & 155 \\
\text { G1 } & 109 \\
\text { G2 } & 46\end{array}$ & $\begin{array}{r}117 \\
89 \\
28\end{array}$ & $\begin{array}{l}75,48 \\
81,65 \\
60,87\end{array}$ & $\begin{array}{l}38 \\
20 \\
18\end{array}$ & $\begin{array}{l}24,52 \\
18,35 \\
39,13\end{array}$ \\
\hline 3 & $\begin{array}{lr}\text { Total } & 198 \\
\text { G1 } & 94 \\
\text { G2 } & 104\end{array}$ & $\begin{array}{c}151 \\
70 \\
81\end{array}$ & $\begin{array}{l}76,26 \\
74,47 \\
77,88\end{array}$ & $\begin{array}{l}47 \\
24 \\
23\end{array}$ & $\begin{array}{l}23,74 \\
25,53 \\
22,12\end{array}$ \\
\hline 4 & $\begin{array}{lr}\text { Total } & 173 \\
\text { G1 } & 144 \\
\text { G2 } & 29\end{array}$ & $\begin{array}{c}127 \\
107 \\
20\end{array}$ & $\begin{array}{l}73,41 \\
74,31 \\
68,97\end{array}$ & $\begin{array}{r}46 \\
37 \\
9\end{array}$ & $\begin{array}{l}26,59 \\
25,69 \\
31,03\end{array}$ \\
\hline 5 & $\begin{array}{lr}\text { Total } & 110 \\
\text { G1 } & 53 \\
\text { G2 } & 57\end{array}$ & $\begin{array}{l}97 \\
49 \\
48\end{array}$ & $\begin{array}{l}88,18 \\
92,45 \\
84,21\end{array}$ & $\begin{array}{r}13 \\
4 \\
9\end{array}$ & $\begin{array}{r}11,82 \\
7,55 \\
15,79\end{array}$ \\
\hline TOTAL & 856 & 658 & 76,87 & 198 & 23,13 \\
\hline
\end{tabular}

\section{Conclusions}

Cette recherche permet déjà de tirer quelques conclusions quant au type de communication qui s'instaure dans les institutions scolaires visitées.

86

Tout d'abord l'enquête a mis en évidence I'utilité qu'il y a à distinguer au sein du milieu scolaire deux situations au moins, ayant leurs caractéristiques linguistiques propres.

A l'intérieur de chaque situation, nous constatons selon ces critères utilisés une uniformité, voire même une homogénéité absolument remarquables, d'établissement en établissement, en ce qui concerne les différents cours de grammaire et les différents cours de vocabulaire. Les performances d'élèves 
seraient avant tout en rapport avec le contenu du cours et le type de pédagogie pratiqué dans l'enseignement de chaque matière : en milieu scolaire ces deux facteurs conditionnent bien davantage les performances linguistiques des enfants que la personne de leur interlocuteur principal, le maître, dont l'individualité, diraiton, serait subordonnée aux objectifs et au poids de mécanismes d'échanges hautement institutionnalisés prescrits pour son cours. On pourrait même parler d'un certain effacement de l'apport individuel de l'enseignant devant les exigences des programmes et/ou la formation pédagogique et l'expérience antérieure du maître au sein de l'institution dans laquelle il est appelé à travailler.

A rappeler aussi que la possibilité ouverte à chaque enfant d'accéder à la parole et la manière dont il peut le faire varient de façon précise et déterminée d'une situation à l'autre. Cette participation plus ou moins grande des élèves, avec des variations syntaxiques extrêmement importantes entre les situations, nous amène à rejeter toute théorie qui d'une part ferait des enseignants les agents d'un système scolaire toujours favorable à la bourgeoisie et qui exclurait les prises de parole des enfants non favorisés. Tout autant que nous rejetons l'hypothèse d'une déficience linguistique, présente dans toute situation chez les enfants défavorisés.

L'on a bien vu que d'une part certains enseignants font montre d'un effort particulier en vocabulaire pour introduire les enfants dits « défavorisés » dans le circuit de la communication, et que d'autre part tous les élèves, quel que soit leur milieu socioculturel d'origine, s'expriment en grammaire de manière identique d'un point de vue syntaxique. Néanmoins les différences inter-classes sociales entre les performances des élèves (au niveau de la syntaxe en vocabulaire, au niveau des stratégies d'intervention en grammaire) obligent à conclure que le facteur socioculturel ne peut être séparé des autres aspects de l'échange en milieu scolaire. Nous rappelons néanmoins encore que les variations intra-groupe social sont suffisamment importantes pour que l'on soit amené à conclure à un type d'échange où sont en jeu plusieurs facteurs : contenu, milieu socioculturel des locuteurs, pédagogie, variations individuelles. Ce qui exigerait un modèle d'analyse portant davantage sur les phénomènes d'interaction maître-enfant.

Certains des phénomènes ici notés semblent se retrouver dans beaucoup de situations (en particulier, à des degrés divers, la répartition inégale des prises de parole). La répartition quantitative de ces phénomènes et d'autres aspects, comme la relation entre prises de parole des enfants en réponse aux enfants et prises de parole des enfants en réponse aux adultes sont sans doute davantage sous la dépendance des traits particuliers de la situation. De ce point de vue, il importerait de comparer cette situation à d'autres, où les enfants seraient plus réellement obligés de collaborer. D'autre part s'impose la comparaison des données recueillies en France et de celles recueillies dans d'autres pays. Tout observateur étranger est en effet immédiatement frappé par l'uniformité des contenus et, par là, dans une certaine mesure, des stratégies pédagogiques caractéristiques de la situation française. 